\title{
Effects of Purinergic Stimulation on Ciliary Beat Frequency and Chloride Secretion in Sinusitis
}

\author{
In-Sang Kim, MD, PhD; Chae-Seo Rhee, MD, PhD; Jun Ho Lee, MD, PhD; Jeong-Hwa Heo, BSc; \\ Jerry Park, BSc; Chul Hee Lee, MD, PhD
}

Objectives: We aimed to identify the functional abnormality of sinusitis-affected mucosa by observing the responsiveness of the mucosa to purinergic stimulation after the onset of sinusitis and during the recovery period. We also aimed to identify possible beneficial effects of purinergic agonists on sinusitis.

Methods: A rabbit sinusitis model was developed by blocking maxillary ostia. Sinus mucosae were harvested immediately and 1 and 4 weeks after reopening the ostia. We measured chloride secretion and ciliary beat frequencies responding to purinergic stimulation.

Results: The increases of ciliary beat frequency by adenosine triphosphate $(100 \mu \mathrm{mol} / \mathrm{L})$ were $3.2 \% \pm$ $8.5 \%, 7.9 \% \pm 2.3 \%$, and $12.2 \% \pm 1.9 \%$ immediately after establishment of sinusitis and 1 week and 4 weeks after reopening of ostia, respectively. Chloride secretion stimulated by adenosine triphosphate also showed gradual increase during the recovery period. Grossly, the mucosae appeared to have normalized in $80 \%$ (4 of 5 ) after 4 weeks; however, functional and microscopic improvements were still incomplete.

Conclusions: Mucosal functions, assessed by increase of ciliary activity and ion secretion by purinergic stimulation, and microscopic findings showed gradual but incomplete recovery after 4 weeks of recovery, in contrast to the gross normalization. $\mathrm{Pu}$ rinergic agonists may have beneficial effects on sinusitis by stimulating decreased ciliary motility and chloride secretion in sinusitis.

From the Department of Otorhinolaryngology (I.-S.K.), College of Medicine, Dankook University, Cheonan, Korea; and the Department of Otorhinolaryngology (C.-S.R., J.H.L., J.-H.H., J.P., C.H.L.), Seoul National University College of Medicine, Seoul, Korea. 6, 2007 .

Editor's Note: This Manuscript was accepted for publication April

This work was supported by a Seoul National University Hospital grant (\#03-2005-028-0).

Send correspondence to Dr. Chae-Seo Rhee, Associate Professor, Department of Otorhinolaryngology, Seoul National University Hospital, 28 Yongon-Dong, Chongno-Gu, Seoul 110-744, Korea. E-mail: csrhee@snu.ac.kr

DOI: 10.1097/MLG.0b013e31806911ce
Key Words: Sinusitis, purinergic receptors, chloride channels, mucociliary clearance.

Laryngoscope, 117:1677-1682, 2007

\section{INTRODUCTION}

Rhinosinusitis is an acute or chronic inflammation of paranasal sinuses and the nasal airway tract. When inflamed, mucus secretion is increased from sinus mucosa, and mucociliary transport is reduced, resulting in a thick, profuse discharge. ${ }^{1}$

Rhinosinusitis develops because of impaired mucociliary clearance or obstruction of ostia. The surgical opening of ostia should promote the recovery of sinus epithelium and function..$^{2,3}$

However, reports are contradictory concerning the morphologic changes that occur after the restoration of sinus ventilation. One report concluded that sinus mucosae can be normalized by restoring normal ventilation after endoscopic sinus surgery in patients with chronic rhinosinusitis, whereas others have observed many pathologic findings even 6 months postoperation. ${ }^{4}$ Furthermore, functional changes in sinusitis and their relations with morphologic changes have been little studied.

Increasing evidence indicates that ion channel dysfunction is involved in the pathophysiology of rhinosinusitis. It has been reported that ion transport processes play a role in the determination of the viscoelasticity of maxillary sinus fluid in chronic rhinosinusitis and that mutations in the gene responsible for cystic fibrosis (CF) may be associated with the development of chronic rhinosinusitis in the general population. ${ }^{5,6}$ Airway luminal purinergic receptors are known to play a major role in the regulation of mucociliary clearance and of the fluid homeostasis of airway surface liquid. Thus, purinergic agonists have been studied in terms of their therapeutic potentials in CF, which are characterized by enhanced airway $\mathrm{Na}^{+}$absorption and deficient $\mathrm{Cl}^{-}$transport. Moreover, clinical trials have found that purinergic agonists can counteract ion transport defects in CF. ${ }^{7}$ Purinergic activation has been shown to improve airway surface hydration in $\mathrm{CF}$ and to 
promote other components of mucociliary clearance, such as ciliary beat frequency (CBF) and mucus secretion. ${ }^{8}$

Airway ion transport and mucociliary clearance are components of the defense mechanisms of airways and are closely related functionally. Thus, we used purinergic agonists to investigate the function of sinus mucosa. We observed functional changes of the ion transport system and ciliary activity and examined their responses to purinergic agonists in sinus mucosae over a 4 -week recovery period after the surgical correction of sinusitis. Correlations between functional changes and morphologic alterations of sinus mucosae were observed using an animal model.

\section{METHODS}

\section{Rabbit Sinusitis Model}

New Zealand white rabbits of either sex and a body weight of 1.5 to $2.5 \mathrm{~kg}$ were used in this study. After intramuscular injection of a mixture of ketamine $50 \mathrm{mg} / \mathrm{kg}$ and xylazine 10 $\mathrm{mg} / \mathrm{kg}$, a $2.5-\mathrm{cm}$ long incision was made on a side of the nasal dorsum, exposing the anterior maxillary sinus wall. After partial removal of the bony wall, a sterile synthetic sponge based on cellulose (cellulose $35 \%$, regenerated cellulose $65 \%$, SugoMed, Eschenburg, Germany), which is used for tamponade after ENT operations, was inserted into the sinus cavity to obstruct the natural ostium of the maxillary sinus. This was followed by tight skin closure. Two weeks later, the sponge was removed, and the natural ostium was reopened. Gross purulent discharge was observed in all surgically treated animals.

Animals with sinusitis were divided into three groups, and animals in these groups were sacrificed on the day of reopening of the ostium ( 0 wk group), at 1 week ( 1 wk group), or at 4 weeks after ostium reopening (4 wk group). Rabbits with normal, healthy sinus mucosae (i.e., without intervention) were used as controls. Five rabbits were allocated to each group. Maxillary sinus mucosae were carefully dissected from the bony walls and divided into three pieces for CBF measurement, ion channel analysis, and histopathologic examination. All experiments and procedures were approved by the Institutional Ethical Committee for Animal Experimentation.

\section{Voltage-Sensitive Vibrating Probe Technique}

The vibrating probe technique, which was identical to that previously described, was used to measure extracellular electrical currents, on the epithelial surface, of a steady or slowly changing nature. ${ }^{9}$ A vibrating probe tip of $20 \mu \mathrm{m}$ diameter, located at 30 to $40 \mu \mathrm{m}$ from the epithelial surface, was used to detect voltage changes in the nanovolt range. The maxillary sinus mucosae samples were perfused continuously with physiologic saline containing (in $\mathrm{mM}$ ) $130 \mathrm{NaCl}, 5 \mathrm{KCl}, 1 \mathrm{MgCl}_{2}, 1 \mathrm{CaCl}_{2}, 5$ glucose, and $10 \mathrm{~N}-2$-hydroxyethylpiperazine-N'-2-ethanesulfonic acid (HEPES), pH 7.4. Adenosine 5' -triphosphate (ATP, $100 \mu \mathrm{mol} / \mathrm{L}$, Sigma A-9187, St. Louis), which evoked the robustest responses in mucosa compared with other purinergic agonists in the preliminary study, was used for purinergic stimulation. Dimethyl sulfoxide, $0.1 \%$, used for dilution, had no effect on the short-circuit current on the epithelial surface. ATP was added in the presence of amiloride to attenuate epithelial $\mathrm{Na}^{+}$absorption.

\section{CBF Measurement}

Ciliary movement was observed using an immersion microscope at a magnification of $\times 600$. A digital charge-coupled device camera (XC-HR50, Sony Co., Japan) capable of operating at 60 full frames per second speed transferred images to a computer equipped with a frame grabber (PCVisionplus, Dalsa Co., St.
Laurent, Canada). Ciliary movement was analyzed using software based on fast-Fourier transform. ${ }^{10}$

\section{Histopathologic Examination}

Mucosal samples for light microscope were fixed in 3.7\% formaldehyde for 24 hours. Tissue samples were then dehydrated and embedded in paraffin. Sections $(6 \mu \mathrm{m})$ were stained with hematoxylin-eosin. Samples for scanning electron microscope (SEM) were prefixed in $2.5 \%$ glutaraldehyde buffer $(\mathrm{pH}=7.4)$, postfixed with $1 \%$ osmium tetroxide solution, and after dehydration using graded ethanol series were dried and coated with platinum. The mounted specimens were observed by SEM (Hitachi S-4300, Tokyo, Japan).

\section{Data Analysis}

Data are expressed as means $\pm \mathrm{SE}$ of mean $(\mathrm{n}=$ number of animals). Statistical significance $(P<.05)$ was determined using the paired $t$ test and analysis of variance. SPSS 11.0 (SPSS, Inc., Chicago, IL) was used throughout.

\section{RESULTS}

\section{Effects of Purinergic Agonists on Normal Mucosal CBFs and Chloride Secretion}

In the normal control group, ATP induced strong and robust increase of CBFs. One hundred micromoles of ATP strongly stimulated $\mathrm{CBF}$, with an average increase of $30.1 \% \pm 2.6 \%(\mathrm{n}=5, P<.05)$. CBFs increased immediately after exposure to ATP.

ATP also induced a rapid increase in anion current, and this effect was blocked by diphenylamine-2,2'dicarboxylic acid (DPC) in the control group mucosa $(\mathrm{n}=$ 5), indicating that ATP induced a DPC-sensitive chloride current, probably via cystic fibrosis transmembrane conductance regulator (CFTR) (Fig. 1). Changes of current density showed initial positive peaks and were followed by a rapid decrease (increased $\mathrm{Cl}^{-}$current) and a gradual increase (decreased $\mathrm{Cl}^{-}$current) (Fig. 2). Moreover,

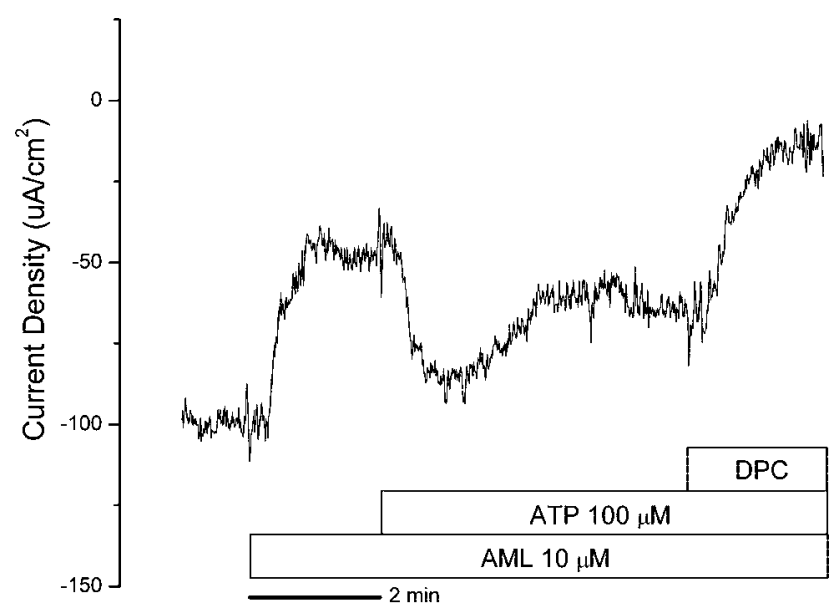

Fig. 1. Anion current changes by adenosine $5^{\prime}$-triphosphate (ATP) and diphenylamine-2,2'-dicarboxylic acid (DPC). ATP induced a rapid increase in anion current, and this effect was blocked by DPC in the control group $(n=5)$, suggesting that ATP induced a DPCsensitive chloride current, probably via cystic fibrosis transmembrane conductance regulator (representative data). 


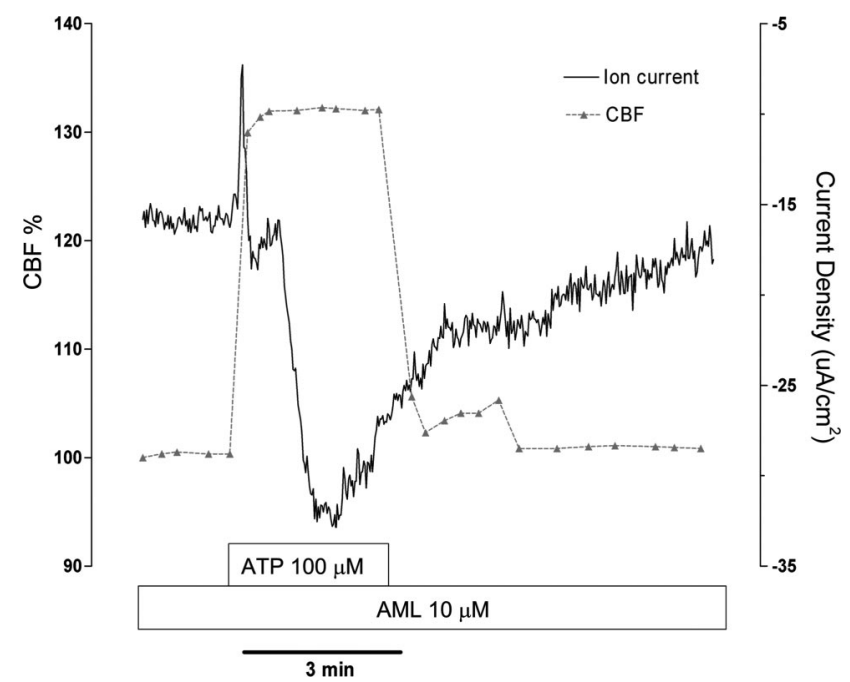

Fig. 2. Ciliary beat frequency (CBF) and ion current changes induced by adenosine 5 '-triphosphate (ATP) in the control group. CBF peaked immediately after exposure to ATP, with a rapid increase in chloride current. Chloride current gradually returned to baseline during purinergic stimulation; however, CBF showed a sustained response (representative data).

rapid increases in chloride current coincided with rapid $\mathrm{CBF}$ increases.

\section{Changes of CBF and Chloride Secretion During Recovery From Sinusitis}

ATP was used to evaluate functional changes in sinusitis mucosa because it was found to induce a robust strong response in all groups. The response patterns of concomitant rapid increases in $\mathrm{CBF}$ and in chloride secretion were observed in all groups. However, the response of $\mathrm{CBF}$ to ATP was strongest in the control group and weakest in the 0 week group. In the 0 week group, ATP increased $\mathrm{CBF}$ by $3.2 \% \pm 8.5 \%$; in the 1 week group, this

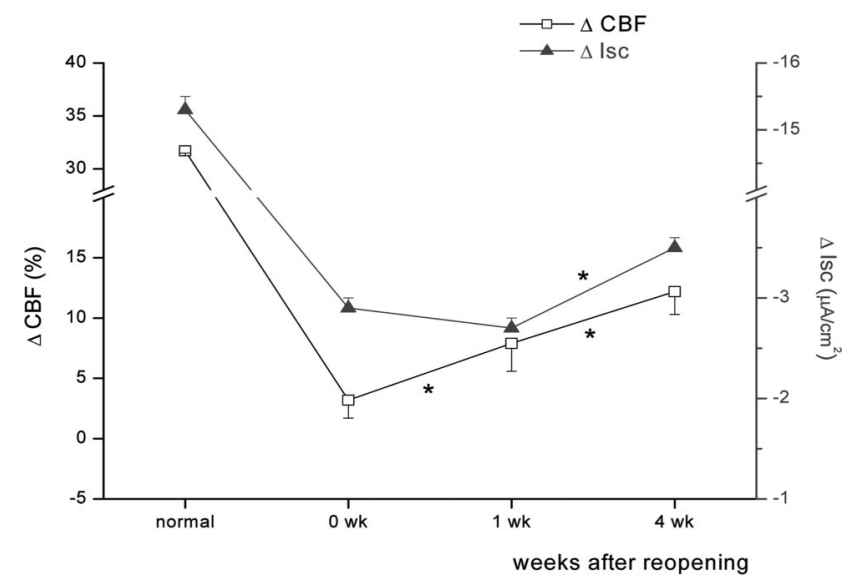

Fig. 3. Response to adenosine 5'-triphosphate (ATP) according to recovery period. Effect of increasing ciliary beat frequency (CBF) and chloride current by ATP was strongest in normal mucosa. Response was weakest in the 0 week group. The four week group showed statistically significant recoveries in both chloride channel activity and $\operatorname{CBF}(P<.01)$. was $7.9 \% \pm 2.3 \%$, which represented a slight, although nonsignificant, recovery. However, the 4 week group showed further significant recovery by $12.2 \% \pm 1.9 \%(P<$ .01 , vs. 0 and 1 wk groups).

Chloride secretion induced by ATP was also greatest in the control group and weakest in the 0 week group. When the epithelial surface current density change ( $\Delta$ Isc) induced by ATP in the control group was set as $100 \%$ $\left(-15.3 \pm 0.2 \mu \mathrm{A} / \mathrm{cm}^{2}\right), \Delta \mathrm{Isc}$ by ATP in the 0,1 , and 4 week groups were $18.9 \% \pm 0.7 \%\left(-2.9 \pm 0.1 \mu \mathrm{A} / \mathrm{cm}^{2}\right), 17.6 \% \pm$ $0.7 \%\left(-2.7 \pm 0.1 \mu \mathrm{A} / \mathrm{cm}^{2}\right)$, and $22.9 \% \pm 0.7 \%(-3.5 \pm 0.1$ $\mu \mathrm{A} / \mathrm{cm}^{2}$ ), respectively. The 0 and 1 week groups showed no significant difference in $\Delta \mathrm{Isc}$ by ATP, but the 4 week group showed a significant difference versus the 0 and 1 week groups $(P<.01)$.

Ciliary activity recovery was matched by ion channel activity recovery (Fig. 3 ). The 0 week group showed profound loss of reactivity to ATP, and in some specimens, this group showed almost no or a paradoxical response to purinergic agonists (e.g., a decrease in $\mathrm{CBF}$ and chloride secretion), suggesting severe functional mucosal deterioration. The 4 week group showed obvious $\mathrm{CBF}$ and ion channel recovery, but these were still below the normal level.

\section{Morphologic Changes in Sinus Mucosa}

Five randomly selected fields per sample were examined under an SEM at $\times 600$. Ciliated cell numbers were determined semiquantitatively as follows: when $75 \%$ or more of a field was covered with ciliated epithelium, it was awarded 3 points; when $25 \%$ to $75 \%, 2$ points; when less than $25 \%, 1$ point; and when ciliated epithelium was absent, 0 points. The score for normal mucosa was $2.8 \pm 0.4$, and for the 0,1 , and 4 week groups were $0.4 \pm 0.3,0.5 \pm$ 0.4 , and $1.7 \pm 0.5$, respectively ( $\mathrm{n}=5$ for each group). In the 0 week group, profound epithelial damages were observed (i.e., ciliary loss, ciliary disorientation, deformed short microvilli, thick mucus, and nonciliated areas). The 1 week group showed marked nonciliated areas and thick mucus plugs, like the 0 week group, but short cilia were often observed, indicating ciliogenesis. In the 4 week group, marked ciliary regeneration was observed. However, individual cilium appeared weak and pliable and showed some degree of disorientation, and abnormally short cilia were often observed. Some thick mucus was still observed on epithelial surfaces, indicating increased mucus secretion (Fig. 4).

Grossly, the sinus mucosae of the control group appeared thin. The 0 week group showed gross purulent discharge and marked mucosal thickening in all animals ( 5 of 5). On the other hand, the 1 week group showed mucosal thickening with pus in one animal, the others showing thickening only. The 4 week group appeared grossly normal in four of the five animals, although one animal had an edematous thickened mucosa.

Samples were scored by degree of inflammatory cell infiltration as determined under an optical microscope (i.e., 0 for no inflammatory cell infiltration, 1 for scant infiltration, 2 for obvious infiltration, and 3 for severe infiltration throughout the mucosal layer). The scores obtained were $0.0 \pm 0.0$ for normal mucosa, $2.6 \pm 0.5$ for the 

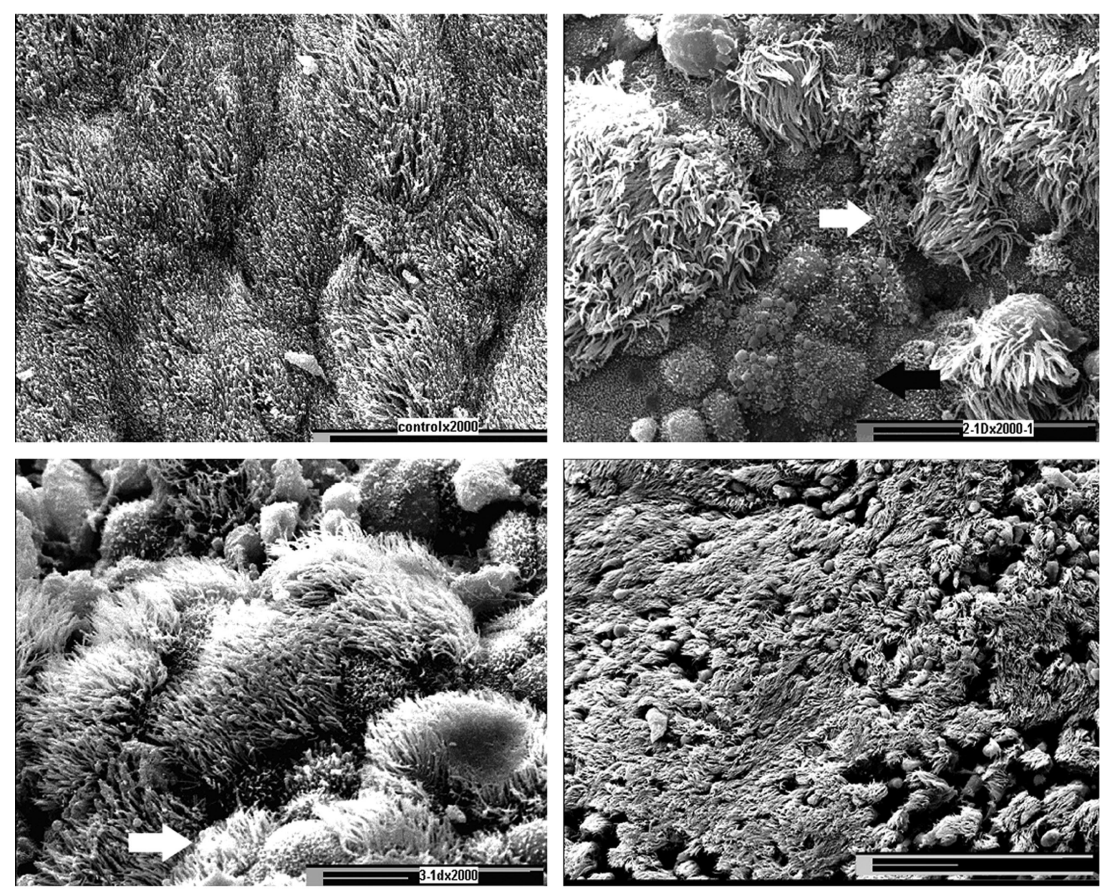

Fig. 4. Scanning electron microscope findings (magnification, $\times 2,000$ ). Normal mucosa (top left). Zero week group (top right). Profound epithelial damage with deformed microvilli (white arrow) and nonciliated areas (black arrow). One week group (bottom left). Short cilia (white arrow) were often observed, indicating ciliogenesis. The four week group showed marked ciliary regeneration (bottom right).

0 week group, $2.4 \pm 0.8$ for the 1 week group, and $1.2 \pm 0.4$ for the 4 week group. Other pathologic changes such as detachment of epithelial ciliated layer, goblet cell hyperplasia, increased submucosal inflammatory cells, submucosal gland hyperplasia, submucosal edema, basal cell hyperplasia, and basement membrane thickening were evident in the 0 week group. These abnormal changes also showed gradual decreases in the 1 and 4 week groups. However, abnormal findings such as increased inflammatory cells, submucosal gland hyperplasia, and basal cell hyperplasia were still observed after 4 weeks of recovery (Fig. 5).

\section{DISCUSSION}

The characteristics of receptors and other components of the nucleotide signaling system regulating muco- ciliary clearance may be of fundamental importance in the treatment of rhinosinusitis, CF, and other airway diseases. The findings of the present study suggest that purinergic agonists have beneficial effects on rhinosinusitis by activating the ion channel and enhancing mucociliary activity. The results also show that functional deterioration caused by rhinosinusitis was not completely recovered 4 weeks after ostium reopening. Moreover, although sinus mucosal morphology had normalized grossly, abnormal microscopic changes in mucosa were observed even after 4 weeks of recovery.

\section{Effects of Purinergic Agonists on Ciliary Activity}

Pharmacologically, $\mathrm{P}_{2} \mathrm{Y}_{2}$ is the major nucleoside triphosphate purinoceptor that regulates the ciliary activity of airway epithelial cells, and ATP primarily acts on
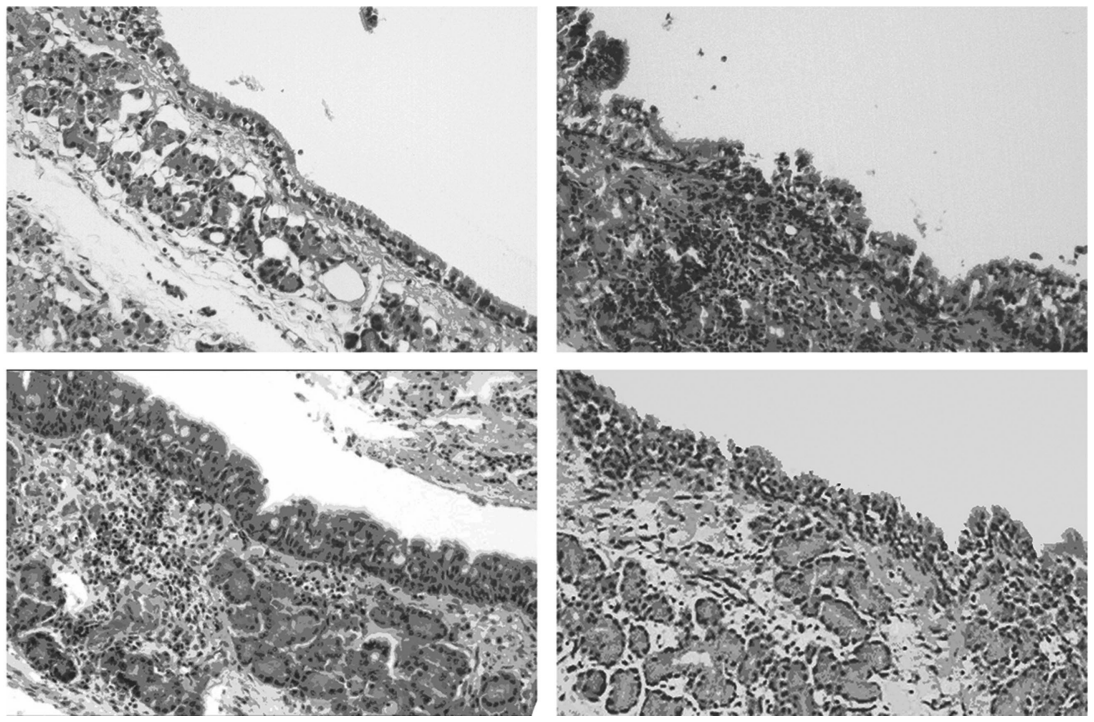

Fig. 5. Hematoxylin-eosin staining (magnification, $\times 200$ ). Normal mucosa (top left). Zero week group showing severe inflammation with epithelial disruption, goblet cell hyperplasia, etc. (top right). One week group (bottom left). Slight improvement of inflammation. Four week group (bottom right). Abnormal findings, such as increased inflammatory cells and submucosal gland hyperplasia, were still observed. 
$\mathrm{P}_{2} \mathrm{Y}_{2}$ receptor. In our preliminary study, uridine-5'diphosphate (UDP) $(100 \mu \mathrm{mol} / \mathrm{L})$ increased CBF by $8.0 \% \pm$ $1.9 \%(\mathrm{n}=5)$, and ATP $(100 \mu \mathrm{mol} / \mathrm{L})$ and uridine-5'triphosphate (UTP) $(100 \mu \mathrm{mol} / \mathrm{L})$ increased CBF by $30.1 \% \pm$ $2.6 \%(\mathrm{n}=5)$ and $30.0 \% \pm 2.1 \%(\mathrm{n}=5)$, respectively, demonstrating that the $\mathrm{P}_{2} \mathrm{Y}_{2}$ receptor is the major CBF regulator in rabbit sinus mucosa. For other purinergic agonists, $100 \mu \mathrm{mol} / \mathrm{L}$ of adenosine-5'-diphosphate (ADP) increased CBF by $6.6 \% \pm 1.3 \%(\mathrm{n}=5)$, and adenosine 100 $\mu \mathrm{mol} / \mathrm{L}$ increased CBF by $7.4 \pm 1.9 \%(\mathrm{n}=5)$. In this study, chloride currents gradually returned to baseline during purinergic stimulation, which suggests receptor desensitization. However, CBFs showed a more sustained response after an initial rise and maintained this elevated level. This observation suggests the presence of mechanisms other than increased chloride secretion for maintaining an elevated CBF. A number of mechanisms have been proposed for prolonged CBF activation even after short-term purinergic stimulation, such as ATP-induced activation of nitric oxide synthase pathways. ${ }^{11}$

\section{Activation of Defective Chloride Secretion in Sinusitis by Purinergic Agonists}

In sinusitis, secretions of excessive viscosities that are poorly mobilized by cilia are a typical finding. ${ }^{1}$ It has been reported that the ion transport process plays a role in determining the viscoelasticity of maxillary sinus fluid in chronic sinusitis. ${ }^{9}$ Stimulation of ion transport and the accompanying water flux provides a natural defense system that functions to effectively wash away noxious stimuli in the settings of cellular damage or inflammation. In this experiment, the functional changes in ion transport should be a consequence of sinusitis. However, it also could be a predisposing factor for sinusitis.

The experimental inhibition of CFTR was found to reduce liquid secretion substantially and cause plugging of submucosal gland ducts with abnormally thick mucinous secretions. ${ }^{12}$ It has also been suggested that CFTR is involved in the pathogenesis of sinusitis. Acquired alterations in CFTR expression and function under physiologically stressful conditions, such as upper respiratory infection or mutations in the gene responsible for $\mathrm{CF}$, may be associated with the development of chronic rhinosinusitis. ${ }^{6,13}$

Activation of chloride transport by purinergic agonists, with or without concurrent treatment with amiloride or amiloride analogs, offers a means of bypassing defects in CFTR-regulated chloride transport. Moreover, a phase I clinical trial on an UTP analogue, INS37217, in $\mathrm{CF}$ patients produced promising results. ${ }^{14}$

If ATP depletion occurs, for example in ischemia, it induces a loss of apical CFTR expression and chloride secretory function, which is followed by marked alterations in junctional complexes and the cytoskeletal structure of respiratory epithelium. ${ }^{15}$ In sinusitis, oxygen tension drops within the sinus cavity, which in turn creates the ischemic conditions that may induce ATP depletion and the functional deterioration of ion channels. Considering the results of this study and of previous reports, it appears that supplementation of purinergic agonists may have beneficial effects on sinusitis, which may have genetic or acquired defects in ion channel functions.

\section{Morphologic and Functional Changes of Mucosa in Sinusitis}

There are some limitations of the animal model. In our rabbit model, we approached the maxillary sinus through the transfacial approach. Using the animal model, in addition to the inherent physiologic and anatomic differences between species, possibilities of iatrogenic injuries, wound infections, and damage to the normal physiologic drainage system are present. Therefore, the conditions may be different in human sinusitis.

One study showed that human sinus mucosa regenerated and returned to normal 6 months postoperatively after functional endoscopic sinus surgery. ${ }^{3}$ However, in another study, many pathologic ultrastructural changes remained in the mucosa 6 months after endoscopic sinus surgery. ${ }^{4}$ In the present study, 4 weeks after restoring normal sinus ventilation, many ultrastructural abnormalities were observed in mucosae, and incomplete microscopic recovery was correlated with incomplete functional recovery. However, macroscopic findings differed from microscopic and functional findings. In macroscopic examinations, mucosae were thin and appeared grossly normal in four of five samples in the 4 week group. In contrast, the 0 and 1 week groups showed marked mucosal thickening with or without pus. These observations suggest that clinical normalization of sinus assessed by postoperative endoscopic and radiographic examinations after sinus surgery may not guarantee microscopic or functional mucosal recovery. Therefore, longer, cautious postoperative follow-up and management may be necessary even after endoscopic or radiographic normalization because incomplete recovery may predispose the mucosa to reinfection or disease relapse.

Purinergic agonists may be delivered in aerosol form to the nasal cavity or to sinus cavities opened by sinus surgery. In individuals with an underlying genetic dysfunction of ion secretion in sinus mucosa, the administration of purinergic agonists may be considered an alternative treatment option. However, other adjuvant treatment modalities should be used in combination because chloride secretion and ciliary activity responding to purinergic agonists may be markedly attenuated in sinusitis mucosae. Moreover, many nonciliated areas, which cannot respond to purinergic agonists, may be present in diseased mucosae. Therefore, conventional treatment modalities, such as antibiotics, mucolytics, nasal irrigation, and surgery are likely to be important. Purinergic agonists are more likely to be beneficial when used as adjunctive treatments, especially during the early stage or recovery period of sinusitis.

We used ATP in this study for purinergic stimulation. However, the metabolites of ATP cause acute bronchoconstriction in human asthmatics, and infusion of ATP causes systemic hypotension. Another potent triphosphate nucleotide, UTP, is known to be relatively safe; however, it has a limited half-life on airway surfaces. Therefore, for the therapeutic use of purinergic agonists in sinusitis, future studies on animals and humans with appropriate analogs with enough half-life and minimal toxicity will be necessary. 


\section{CONCLUSIONS}

Functional deterioration by sinusitis gradually recovers with time. However, in this study, functional and microscopic changes were found to recover incompletely 4 weeks after the restoration of normal sinus ventilation and drainage, in contrast to gross normalization. This implies that clinical normalization of mucosa may not reflect the functional and microscopic normalization. Furthermore, the administration of purinergic receptor agonists was found to have beneficial effects on sinusitis mucosa by enhancing two major components of mucociliary transport: ciliary activity and mucosal ion secretion.

\section{BIBLIOGRAPHY}

1. Wilson R, Sykes D, Currie D, et al. Beat frequency of cilia from sites of purulent infection. Thorax 1986;41:453-458.

2. Kennedy D, Zinreich J, Rosenbaum A, et al. Functional endoscopic sinus surgery: theory and diagnostic evaluation. Arch Otolaryngol Head Neck Surg 1985;111:576-582.

3. Keles N, Ahishali B, Illicali C, et al. Evaluation of ultrastructural changes in paranasal sinus mucosa after functional endoscopic sinus surgery in patients with chronic rhinosinusitis. Am J Rhinol 2001;15:225-229.

4. Toskala E, Rautiainen M. Electron microscopy assessment of the recovery of sinus mucosa after sinus surgery. Acta Otolaryngol 2003;123:954-959.

5. Takeuchi K, Suzumura E, Hirata K, et al. Role of transepithelial ion transport as a determinant of mucus viscoelasticity in chronic inflammation of the maxillary sinus. Acta Otolaryngol 1991;111:1133-1138.

6. Wang X, Moylan B, Leopold DA, et al. Mutation in the gene responsible for cystic fibrosis and predisposition to chronic rhinosinusitis in the general population. JAMA 2000;284: 1814-1819.

7. Bennett W, Olivier K, Zeman K, et al. Effect of uridine $5^{\prime}$ triphosphate plus amiloride on mucociliary clearance in adult cystic fibrosis. Am J Respir Crit Care Med 1996;153: 1796-1801.

8. Morse D, Smullen J, Davis C. Differential effects of UTP, ATP and adenosine on ciliary activity of human nasal epithelial cells. J Physiol Cell Physiol 2001;280:C1484-1497.

9. Marcus DC, Shipley AM. Potassium secretion by vestibular dark cell epithelium demonstrated by vibrating probe. Biophys J 1994;66:1939-1942.

10. Yi WJ, Park KS, Min YG, et al. Distribution mapping of ciliary beat frequencies of respiratory epithelium cells using image processing. Med Biol Eng Comput 1997;35: 595-599

11. Lieb T, Frei C, Frohock J. Prolonged increase in ciliary beat frequency after short-term purinergic stimulation in human airway epithelial cells. J Physiol 2002;538:633-646.

12. Trout L, King M, Feng W. Inhibition of airway liquid secretion and its effect on the physical properties of airway mucus. Am J Physiol Lung Cell Mol Physiol 1998;18: L258-263.

13. Pruliere-Escabasse V, Fanen P, Dazy AC, et al. TGF-beta 1 downregulates CFTR expression and function in nasal polyps of non-CF patients. Am J Physiol Lung Cell Mol Physiol 2005;288:L77-83.

14. Kunzelmann K, Mall M. Pharmacotherapy of the ion transport defect in cystic fibrosis: role of purinergic receptor agonists and other potential therapeutics. Am J Respir Med 2003;2:299-309.

15. Brezillon S, Zahm J, Pierrot D. ATP depletion induces a loss of respiratory epithelium functional integrity and downregulates CFTR expression. J Biol Chem 1997;272: $27830-27838$ 